\title{
Preliminary experience with tacrolimus in managing Henoch-Schönlein purpura featuring extrarenal symptoms refractory to conventional therapy
}

\author{
Yan Chen \\ The first affiliated hospital of Xiamen University https://orcid.org/0000-0003-4207-3104 \\ Haitao Bai ( $\sim$ baihaitao@163.com) \\ The first affiliated hospital of xiamen university https://orcid.org/0000-0002-6711-7225 \\ Yang Yang \\ The first affiliated hospital of xiamen university \\ Zhulan Lian \\ The first affiliated hospital of xiamen university
}

\section{Short Report}

Keywords: Tacrolimus, Henoch-Schönlein purpura, Extrarenal symptom, Treatment effectiveness

Posted Date: June 2nd, 2020

DOI: https://doi.org/10.21203/rs.3.rs-28459/v1

License: (c) (i) This work is licensed under a Creative Commons Attribution 4.0 International License. Read Full License 


\section{Abstract \\ Background}

Henoch-Schönlein purpura (HSP) is the commonest vasculitis of childhood, affecting multiple organ systems. The extrarenal manifestations are prominent in some HSP cases, for which no standard treatment is available. It is interesting to examine the value of tacrolimus in such cases.

\section{Findings}

Seventeen children received tacrolimus to manage their refractory extrarenal symptoms of HSP in an open-label prospective study. Tacrolimus was administered at daily dose of $0.05-0.15 \mathrm{mg} / \mathrm{kg}$. Tacrolimus was effective in all cases after treatment for 7-21 days. Tacrolimus treatment resulted in complete resolution of extrarenal symptoms in 15 cases, and partial resolution in the remaining 2 cases. During follow-up, skin purpura reoccurred in 2 cases, abdominal pain and loss of follow-up in 1 case each. Drug-related adverse reaction was not observed in any case. Abnormal renal function test was identified in 7 patients by urinary screening tests before tacrolimus therapy, 4 of them received renal biopsy. Only one patient showed evidence of renal impairment during follow-up.

\section{Conclusions}

Tacrolimus works quickly without drug-related adverse reaction in managing the HSP patients featuring recurrent extrarenal symptoms. Our preliminary experience suggests good clinical efficacy and safety of tacrolimus in such offlabel use.

\section{Introduction}

Henoch-Schönlein purpura (HSP) is the commonest vasculitis of childhood. Pathological examination reveals IgA1 deposition in blood vessel walls. Purpuric rashes, gastrointestinal symptoms, swollen and painful joints, and renal involvement are common clinical symptoms of HSP. The extrarenal symptoms such as skin purpura, gastrointestinal symptoms, swollen and painful joints are generally self-limited, for which only symptomatic treatment is required. However, in some cases, the extrarenal symptoms are refractory, such as persistent or recurrent purpuric rashes, swollen and painful joints, and abdominal symptoms. Recurrence of extrarenal symptoms is not only a high-risk factor for renal involvement [1], but also makes the child and his/her family nervous and anxious, and so affects their quality of life [2]. Currently, steroids, gamma globulin, blood purification, various immunosuppressive agents, and biological products are usually tried in clinical practice with inconsistent results.

Tacrolimus (Fk506) is a calcineurin inhibitor [3], showing good compliance when administered orally. It is widely used in the treatment of pediatric patients with renal disease with good clinical outcome. This prospective, observational study aimed to examine the efficacy and safety of tacrolimus in managing HSP patients featuring extrarenal symptoms refractory to conventional therapy.

\section{Methods}

The study protocol and informed consent form were approved by the institutional review board of the First Affiliated Hospital of Xiamen University. A child was enrolled if all the following criteria were satisfied. (1) The diagnostic 
criteria of HSP proposed by European League Against Rheumatism (EULAR) and European Society for Paediatric Nephrology (ESPN) in 2005 [4]. (2) Fail to respond to the evidence-based conventional HSP treatments as recommended by Immunology Study Group, Society of Pediatrics, Chinese Medical Association (CMA) in 2013 [5], evidenced by presence of persistent or recurrent skin purpura, gastrointestinal or articular symptoms, no family history of diabetes mellitus, and with normal glucose tolerance. (3) Informed consent was obtained from the family of the child about the off-label use of tacrolimus. (4) No history of kidney disease. (5) The purpura caused by other diseases was excluded.

Tacrolimus was administered orally every 12 hours. The total daily dose was $0.05-0.15 \mathrm{mg} / \mathrm{kg}$ [6]. Steroids were still used in all cases. Tacrolimus treatment was stopped one week after resolution of extrarenal symptoms (but not in case of purpura nephritis when longer duration of tacrolimus treatment is required), or no response after treatment for 2 weeks.

Trough serum concentration of tacrolimus was determined by enzyme-linked immunosorbent assay after 3 dosing days (reference range 5-10 $\mu \mathrm{g} / \mathrm{L}$ ). The time to extrarenal symptom resolution was recorded until the endpoint (i.e., 60 days after tacrolimus treatment). Urinalysis and urine protein quantification were repeated biweekly in follow-up period to evaluate drug-related adverse reactions and symptom recurrence.

\section{Results}

All the enrolled children $(n=17)$ were inpatients treated in the Department of Pediatrics, the First Affiliated Hospital of Xiamen University during the period from July 2015 to October 2018, including 10 boys and 7 girls (4.25-14 years old). The extrarenal symptoms were present for 28 to 90 cumulative days before initiation of tacrolimus treatment.

Tacrolimus treatment was effective in all the 17 patients, including complete resolution of extrarenal symptoms in 15 cases, partial resolution in the remaining 2 cases (evidenced by recurrent but less severe skin purpura alone) (Table 1). Tacrolimus treatment worked quickly, which was proved by resolution of gastrointestinal and articular symptoms within 1-4 days, resolution of skin purpura within 2-10 days. The treatment specific for extrarenal symptoms lasted 10-21 days. Renal involvement was found in 2 children during tacrolimus treatment, which was characteristic of nephrotic syndrome (Lee IIIb). Tacrolimus was still used to treat HSP nephritis in one of the two children. Persistent proteinuria and microscopic hematuria were found in 2 cases (Lee lla). Repeated quantification of urine protein did not meet the criteria of nephrotic syndrome. Tacrolimus was stopped after resolution of extrarenal symptoms. During follow-up, skin purpura reoccurred in 2 cases, one associated with abdominal pain (one episode of skin purpura) and another loss to follow-up (two episodes of skin purpura), respectively.

Therapeutic drug monitoring was available in 13 of the 17 patients 3 days after oral administration of tacrolimus. Serum tacrolimus concentration was higher than $5 \mathrm{ng} / \mathrm{ml}$ in 7 cases, lower than $5 \mathrm{ng} / \mathrm{ml}$ in 6 cases. The extrarenal symptoms resolved completely in 6 of the 7 children with higher tacrolimus concentration ( $>5 \mathrm{ng} / \mathrm{ml})$. Skin rashes relapsed in one of the 6 patients with lower tacrolimus concentration $(<5 \mathrm{ng} / \mathrm{ml})$. Tacrolimus-related adverse reaction was not found in any of these patients. One patient developed non-convulsive seizure during treatment associated with normal serum tacrolimus concentration. Brain magnetic resonance imaging showed low density signal in bilateral hippocampal region, which was considered neurological involvement of HSP. HSP was complicated with pancreatitis in one child (serum tacrolimus concentration $<5 \mathrm{ng} / \mathrm{ml}$ ). It was considered as a complication of HSP. Serum levels of amylase and lipase were normalized by symptomatic therapy. Imaging study suggested ease of pancreatic edema and relief of abdominal pain. The extrarenal symptoms did not occur again after rechallenge with 
oral tacrolimus. Another patient developed orchitis and recurrent skin rashes on treatment but lost to follow-up after discharge.

Renal involvement was identified in 7 of the 17 children before tacrolimus treatment, evidenced by various degrees of hematuria and/or proteinuria. Four patients received renal biopsy for pathological examination (Lee lla in one case, Lee Ilb in another case, and Lee IIIb in the remaining 2 cases). One child developed hematuria alone during follow-up, but renal biopsy was not available.

\section{Discussion}

HSP is the commonest vasculitis of childhood, affecting the small blood vessels, with annual incidence of 1020/100,000 [7]. HSP is primarily mediated by IgA1 (abnormal glycosylation in the hinge region) deposition in the walls of blood vessels and glomerular mesangium. Therefore, it was renamed as IgA vasculitis (IgAV) at International Chapel Hill Consensus Conference in 2012 [8]. For the HSP patients with prominent refractory extrarenal symptoms, no optimal therapy is available at present time. Steroids, gamma globulin, and blood purification are usually used in clinical practice, but associated with higher incidence of steroids-related side effects, and emergence of resistance to steroids and gamma globulin. Blood purification is invasive and expensive, which is not applicable for the treatment of non-life-threatening HSP. In recent years, several immunosuppressants and biological products such as methotrexate, azathioprine, dapsone, colchicine, and rituximab, have also been reported with some successes [9-13].

Tacrolimus is a potent immunosuppressive agent widely used in pediatric kidney diseases. It works primarily by interfering with calcium-dependent signaling pathway and inhibiting the transcription of $T$ cell activation-related gene and subsequent protein synthesis. Tacrolimus can also inhibit cytokine production and IL-2R expression. It is a dual inhibitor of both cellular and humoral immunity. Bhimma [14] et al found that in 20 children with steroid-resistant focal segmental glomerulosclerosis (FSGS), 85\% achieved complete or partial resolution after tacrolimus plus low dose steroids combination therapy for 12 months. The relapse rate was only $10 \%$ after average follow-up period of 27.5 months. The adverse reactions such as vomiting, anemia, and diarrhea at early stage of oral drug therapy are self-limited, suggesting good treatment safety and efficacy. In a study of steroid-resistant nephrotic syndrome (SRNS), Loeffler [15] found 16 patients switched to tacrolimus due to resistance/dependence $(n=15)$ and intolerable side effects from other therapies $(n=1)$. Tacrolimus showed a complete remission rate of $81 \%$ and a partial remission rate of $13 \%$. The overall response rate was $94 \%$. Tacrolimus is much better than other immunosuppressants (mycophenolate mofetil, cyclophosphamide, and cyclosporine A) in FSGS or SRNS in terms of efficacy and safety profiles.

To our knowledge, tacrolimus has not been reported in treatment of recurrent HSP. It is supposed that the effect of tacrolimus found in our study may be associated with its inhibition of T cell proliferation, and so influencing T-cell dependent B cell differentiation and maturation, which reduces the synthesis and secretion of IgA1. On the other hand, tacrolimus can indirectly block alternative complement pathway, which reduces the formation of immune complex IgA-IgG-C3. Tacrolimus acts through these two pathways to relieve the lesions of small blood vessels [16].

Our study showed that tacrolimus was significantly effective in managing all the 17 HSP patients featuring refractory extrarenal symptoms. The extrarenal symptoms resolved in 15 cases during treatment. The remaining 2 patients achieved partial resolution. Tacrolimus treatment is safe in all cases without any drug-related adverse reaction. This study is observational in design and preliminary experience of our clinical practice. More issues and topics such as duration, dosage, time points for evaluation, reduction of renal involvement, and long-term complications, should be further examined and clarified in clinical practice, and optimally confirmed in randomized controlled clinical trials. 
In summary, the results of this study suggest that for the HSP patients with refractory extrarenal symptoms who fail to respond to conventional therapy, tacrolimus can be considered as a worthwhile treatment option for its quick effect, good outcome, and safety profile.

\section{Abbreviations}

CMA: Chinese Medical Association; ESPN: European Society for Paediatric Nephrology; EULAR: European League Against Rheumatism; FSGS: focal segmental glomerulosclerosis; HSP: Henoch-Schönlein purpura; IgAV: IgA vasculitis; SRNS: steroid-resistant nephrotic syndrome

\section{Declarations}

\section{Compliance with ethical standards}

The study protocol and informed consent form were approved by the institutional review board of the First Affiliated Hospital of Xiamen University. Legal guardian of the participants provided informed consent before study.

\section{Acknowledgments}

The authors would like to thank the family of the children for their cooperation in the study.

\section{Authors' contributions}

The study was designed by $\mathrm{CY}$ and $\mathrm{BH}$. All authors (CY, BH, YY and LZ) were responsible for data collection. All authors had reviewed study data, revised the manuscript and approved the final version for publication.

\section{Funding}

Not applicable.

\section{Consent for publication}

All authors approved the publication of this manuscript.

\section{Conflict of interest}

All authors declare no conflict of interest.

\section{References}

1. Saulsbury FT. Successful treatment of prolonged Henoch-Schönlein purpura with colchicine. Clin Pediatr (Phila). 2009;48:866-8.

2. Saulsbury FT. Clinical update: Henoch-Schonlein purpura. Lancet. 2007;369:976-8.

3. Wu CS, Lan CC, Kuo HY, Chai CY, Chen WT, Chen GS. Differential regulation of nuclear factor-kappa B subunits on epidermal keratinocytes by ultraviolet B and tacrolimus. Kaohsiung J Med Sci. 2012;28:577-85.

4. Ozen S, Ruperto N, Dillon MJ, Bagga A, Barron K, Davin JC, et al. EULAR/PReS endorsed consensus criteria for the classification of childhood vasculitides. Ann Rheum Dis. 2006;65:936-41. 
5. Immunology Study Group, Society of Pediatrics, Chinese Medical Association. Evidence-based recommendations for managing Henoch-Schonlein purpura in children. Zhonghua Er Ke Za Zhi. 2013;51:502-7.

6. Nephrology Study Group, Society of Pediatrics, Chinese Medical Association. Guidelines for managing steroidsensitive, steroid-refractory, and steroid-dependent nephrotic syndrome in children. Zhonghua Er Ke Za Zhi. 2017;55:729-34.

7. Gardner-Medwin JM, Dolezalova P, Cummins C, Southwood TR. Incidence of Henoch-Schönlein purpura, Kawasaki disease, and rare vasculitides in children of different ethnic origins. Lancet. 2002;360:1197-202.

8. Jennette JC, Falk RJ, Bacon PA, Basu N, Cid MC, Ferrario F, et al. 2012 revised International Chapel Hill Consensus Conference Nomenclature of Vasculitides. Arthritis Rheum 2013;65:1-11.

9. Trapani S, Mariotti P, Resti M, Nappini L, de Martino M, Falcini F. Severe hemorrhagic bullous lesions in Henoch Schonlein purpura: three pediatric cases and review of the literature. Rheumatol Int. 2010;30:1355-9.

10. Fotis L, Tuttle PV 4th, Baszis KW, Pepmueller PH, Moore TL, White AJ. Azathioprine therapy for steroid-resistant Henoch-Schönlein purpura: a report of 6 cases. Pediatr Rheumatol Online J. 2016;14(1):37.

11. Saulsbury FT. Henoch-Schönlein purpura in children. Report of 100 patients and review of the literature. Medicine. 1999;78:395-409.

12. Lee KH, Park JH, Kim DH, Hwang J, Lee G, Hyun JS, et al. Dapsone as a potential treatment option for HenochSchönlein Purpura (HSP). Med Hypotheses. 2017;108:42-5.

13. Donnithorne KJ, Atkinson TP, Hinze CH, Nogueira JB, Saeed SA, Askenazi DJ, et al. Rituximab therapy for severe refractory chronic Henoch-Schönlein purpura. J Pediatr. 2009;155:136-9.

14. Bhimma R, Adhikari M, Asharam K, Connolly C. Management of steroid-resistant focal segmental glomerulosclerosis in children using tacrolimus. Am J Nephrol. 2006;26:544-51.

15. Loeffler K, Gowrishankar M, Yiu V. Tacrolimus therapy in pediatric patients with treatment-resistant nephrotic syndrome. Pediatr Nephrol. 2004;19:281-7.

16. Chen O, Zhu XB, Ren P, Wang YB, Sun RP, Wei DE. Henoch Schonlein Purpura in children: clinical analysis of 120 cases. Afr Health Sci. 2013;13:94-9.

\section{Tables}

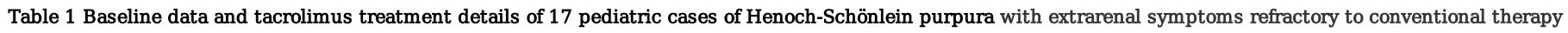

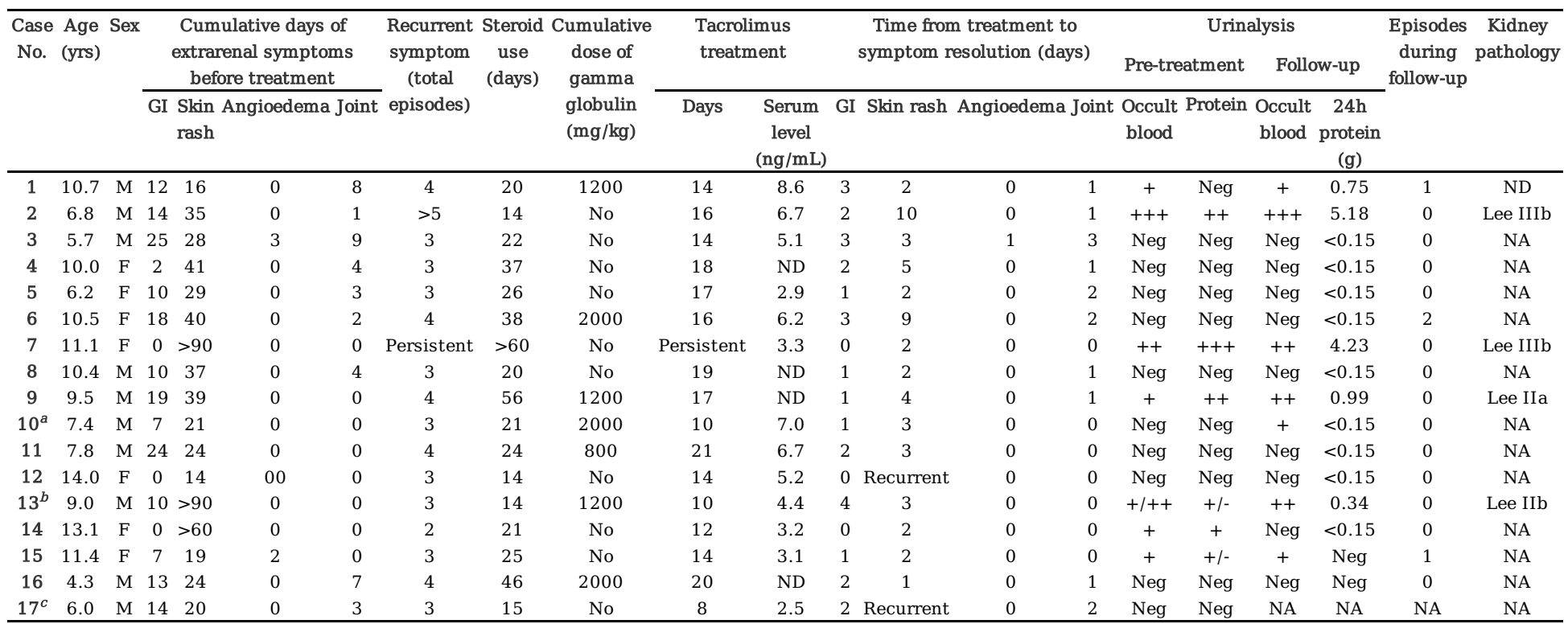




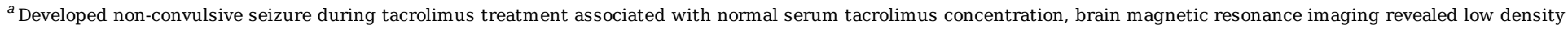
signal in bilateral hippocampal regions, which was considered neurological involvement of Henoch-Schönlein purpura.

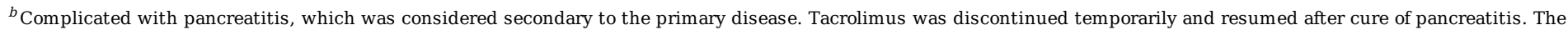
total duration of tacrolimus treatment was 10 days.

${ }^{c}$ Complicated with orchitis, associated with recurrent abdominal pain, and lost to follow-up after discharge.

$F$ female, $G I$ gastrointestinal, $M$ male, $N A$ not available, $N D$ not determined, Neg negative 